MIDPI

MOL2NET, International Conference Series on Multidisciplinary Sciences

sciforum,

\title{
Influence of codon 35 amino acid insertion in HIV-1 protease: insights from molecular dynamics
}

\author{
João P. Luís ${ }^{a}$, Ana I. Mata ${ }^{a}$, Nuno G. Alves ${ }^{a}$, Carlos J. V. Simões ${ }^{a, b}$, João Pereira-Vaz ${ }^{c}$, \\ Daniela C. Vaz ${ }^{a, d}$, Vitor Duque $e^{e}$ Rui M. M. Brito ${ }^{a, b}$ \\ ${ }^{a}$ CQC, Chemistry Department, Faculty of Science and Technology, University of Coimbra, Coimbra, Portugal \\ ${ }^{b}$ BSIM Therapeutics, Instituto Pedro Nunes, Coimbra, Portugal \\ ${ }^{c}$ Laboratory of Molecular Biology, Clinical Pathology Unit, CHUC, Coimbra, Portugal \\ ${ }^{d}$ Health Research Unit, School of Health Sciences, Leiria, Portugal \\ ${ }^{e}$ Infectious Diseases Unit, CHUC, Coimbra, Portugal
}

\begin{abstract}
.
One of the main challenges facing the development of effective anti HIV-1 medicines relates to the high mutation rate of essential enzymes, such as HIV-1 Protease (HIVIPr).[1] Pereira Vaz et al. [2] first reported a threonine insertion at position 35 (E35E_T), in the HIVIPr coding region, among treatment-nä̈ve subtype $C$ infected individuals. Undetectable viral loads were attained after antiretroviral therapy in such individuals, with no associated major mutations, implying null contribution of E35E_T to viral resistance. Interestingly, a new study suggests a potential additive effect of position 35 insertions when in presence of major mutations - ultimately leading to resistance to HIVIPr inhibitors in higher extent. [3]

In order to study the role of the E35E_T insertion in the structure and ligand-binding propensity of HIVIPr, homology models were generated from subtype $B$ and subtype $C$ base sequences, using available X-ray structures corresponding to highest identity sequences as template. Fifty (50)nanoseconds Molecular Dynamics (MD) simulations were then performed for unbound and bound (HIVIPR:darunavir complex) structures of the wild-type form and a single point major mutation variant of HIVIPR - in all cases in presence and absence of E35_T.

Combining simple measurements like the root mean square (RMS) deviations and fluctuations, applied to the whole protein and to its two functional flap regions, with principal component analysis (PCA) of the multiple MD trajectories, we herein contrast the behavior of all systems in attempt to dissect the putative role of E35E_T in the resistance towards HIVIPR inhibitors.
\end{abstract}

\section{References}

[1] Baxter D. J. et al., An Update on HIV-1 Protease Inhibitor Resistance. J AIDS Clin Res. 2016, 7, 581.

[2] Pereira-Vaz J. et al., Detection of the protease codon 35 amino acid insertion in sequences from treatment-naïve HIV-1 subtype C infected individuals in the Central Region of Portugal. J Clin Virol. 2009, 46, 169.

[3] Kozísek M. et al., Ninety-nine is not enough: molecular characterization of inhibitor-resistant human immunodeficiency virus type 1 protease mutants with insertions in the flap region. J Virol. 2008, 82, 5869. 